\title{
A prediction rule for clinical diagnosis of severe acute respiratory syndrome
}

\author{
P.L. Ho*, P.H. Chau\#, P.S.F. Yip\#, G.C. Ooi", P.L. Khong", J.C. Ho", P.C. Wongף, \\ C. Ko", C. Yan" and K.W. Tsang"
}

ABSTRACT: A prospective study was undertaken to identify clinical, radiographical, haematological and biochemical profiles of severe acute respiratory syndrome (SARS) patients.

A prediction rule, which demarcates low from high risk patients for SARS in an outbreak situation was developed. A total of 295 patients with unexplained respiratory illnesses, admitted to Queen Mary Hospital, Hong Kong SAR, China, in March to July 2003, were evaluated for clinical, radiological, haematological and alanine transaminase (ALT) data daily for 3 days after hospitalisation.

In total, 44 cases were subsequently confirmed to have SARS by RT-PCR (68.2\%) and serology (100\%). The scoring system of attributing 11, 10, 3, 3 and 3 points to the presence of independent risk factors, namely: epidemiological link, radiographical deterioration, myalgia, lymphopenia and elevated ALT respectively, generated high and low-risk (total score 11-30 and 0-10, respectively) groups for SARS. The sensitivity and specificity of this prediction rule in positively identifying a SARS patient were 97.7 and $81.3 \%$, respectively. The positive and negative predictive values were 47.8 and $99.5 \%$, respectively.

The prediction rule appears to be helpful in assessing suspected patients with severe acute respiratory syndrome at the bedside, and should be further validated in other severe acute respiratory syndrome cohorts.

KEYWORDS: Coronavirus infection, diagnosis, pneumonia, severe acute respiratory syndrome

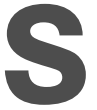
evere acute respiratory syndrome (SARS), caused by a novel coronavirus (SARS$\mathrm{CoV})$, inflicted catastrophe on the economy and immeasurable suffering, particularly among healthcare workers, from February to June 2003. SARS remains a major health threat and this risk is continually aparent, initially by the two single laboratory-acquired cases in Singapore and Taiwan, and more recently by the four community-acquired cases in GUANGDONG [1]. These cases pronounce the need of continued vigilance and vigour in public health measures, and research into the poorly understood pathogenesis, clinical features, radiological appearance and treatment in SARS.

The control of SARS requires early diagnosis and prompt isolation of all suspected cases, as a single index case could result in massive outbreaks [2-5]. Diagnostic criteria proposed by the World Health Organization (WHO) and Center of Disease Control and Prevention of USA (CDC) are predominantly epidemiologically orientated, and understandably rely heavily on microbiological testing for the presence of SARS-CoV $[6,7]$.
The latter comprises primarily of serology and RT-PCR to demonstrate the presence of specific anti-SARS-CoV immunoglobulin (Ig) G and viral RNA in blood and respiratory specimens, respectively $[6,8]$. The use of RT-PCR, despite the initial optimism, does not permit complete confidence at early stages of the disease [5, 9-10], and takes 2-3 days for most microbiology laboratories to complete. Presence of anti-SARS-CoV IgG usually occurs earliest on day 10 of the illness, and serological detection is, therefore, not useful in the initial stages of SARS [5, 11].

The current authors undertook this prospective study, during the 2003 SARS outbreak, to identify clinical, radiographical, haematological and biochemical profiles of SARS patients, and to compare these with those from control patients with non-SARS respiratory illness. The current authors attempted to develop a prediction rule, to demarcate low- from high-risk patients for SARS. They believed this prediction rule should help clinicians in the initial diagnosis and management of potential SARS patients.
AFFILIATIONS

University Depts of *Microbiology, \#Statistics and Actuarial Science, and

'Diagnostic Radiology and Medicine, The University of Hong Kong, Queen Mary Hospital, Hong Kong SAR,

China.

\section{CORRESPONDENCE}

K.W.T. Tsang

Division of Respiratory and Critical

Care Medicine

University Dept of Medicine

The University of Hong Kong

Queen Mary Hospital

Pokfulam

Hong Kong SAR

China

Fax: 85229049443

E-mail: kwttsang@hku.hk

Received:

June 252004

Accepted after revision:

June 022005 


\section{METHODS}

\section{Patient recruitment and study design}

Patients with an unexplained respiratory illness, with fever or history of such in the preceding $48 \mathrm{~h}\left(>38^{\circ} \mathrm{C}\right)$, namely those with upper (rhinorrhea, blocked nose or sore throat) or lower (cough, sputum production or dyspnoea) respiratory symptoms were admitted to the isolation wards of Queen Mary Hospital, Hong Kong, China, for further evaluation and management during the 2003 SARS outbreak. A final diagnosis of SARS was made when patient had fever $\left(\geqslant 38^{\circ} \mathrm{C}\right)$, respiratory symptoms, a four-fold increase in anti-SARS-CoV IgG in acute and convalescence serum [5, 11, 12], and/or detection of SARS-CoV RNA [6, 8].

The derivation cohort comprised of 295 patients each with an unexplained respiratory illness admitted to isolation wards in Queen Mary Hospital, between 17th March and 20th July 2003 [11]. The standard antimicrobial regimen was i.v. cefepime plus oral clarithromycin or i.v. levofloxacin in the presence of penicillin allergy for 10-14 days [2, 11].

\section{Radiographical, haematological, biochemical and microbiological evaluation}

A chest radiograph, complete blood counts, alanine transaminase (ALT) and renal function tests were performed daily [13]. Chest radiographs were scored according to the per cent of lung involvement in each lung $(0=$ normal, $1=10 \%, 2=20 \%$, $10=100 \%$ involvement). Summation of scores from both lungs provided the radiographical score for a particular chest radiograph for each patient. A radiographic progress was regarded as improved, unchanged or deteriorated if the radiographical score (within $5 \%$ ) on day 3 was $<$, equal to, or $>$ that derived on day 1 , respectively [13]. Symptoms, travel and contact history were prospectively obtained by infection control nurses with a standardised questionnaire. An epidemiological link was defined as a history of contact with a SARS patient, or visiting a hospital with nosocomial SARS outbreak, in the 10 days preceding disease onset. Microbiological tests comprised blood culture, sputum bacterial culture, and nasopharyngeal aspirate for common respiratory viral antigens (influenza, parainfluenza, respiratory syncytial virus and adenovirus) and viral culture.

\section{Data analysis and predictive rule development}

Logistic regression models were applied to identify parameters independently associated with SARS [14]. The following parameters were entered for analysis: history of epidemiological link, myalgia and pneumonic symptoms at presentation (presence or otherwise for cough, sputum production, dyspnoea and chills), temperature $\geqslant 38^{\circ} \mathrm{C}$ or history of such in the previous $48 \mathrm{~h}$, upper respiratory tract symptoms (any of rhinorrhoea, blocked nose or sore throat), and gastrointestinal symptoms (any of vomiting, nausea, abdominal pain or diarrhoea). Abnormal laboratory results (presence or otherwise in the first 3 days for thrombocytopenia i.e. platelet count $<150 \times 10^{9} \cdot \mathrm{L}^{-1}$ ), leukocytosis (total leukocyte count $\left.>11 \times 10^{9} \cdot \mathrm{L}^{-1}\right)$, lymphopenia $\left(<1.5 \times 10^{9} \cdot \mathrm{L}^{-1}\right)$, and elevation of $\operatorname{ALT}\left(>53 \mathrm{U} \cdot \mathrm{L}^{-1}\right)$ were also identified for each patient $[2,11]$. A radiographic response was also assessed for each patient by comparing radiographs between day 1 and day 3. A stepwise procedure was used in the selection of independently significant predictors adjusted for other predictors $(\mathrm{p}<0.05)$.

Based on the fitted model, the fitted probabilities could reflect how likely the case would be a SARS case. The current authors followed the approach described by FINE et al. [15] on assessment of severity of community-acquired pneumonia, to use score in place of probability in the prediction rule model. To generate a simple-integer point score, the fitted regression coefficients of the final logistic model were rescaled by dividing their values by the smallest fitted coefficient and rounded to the nearest integer, which retained the relative size of effects of each of the risk factors. For each binary risk factor, the best condition is defined to be absence of risk factor; for radiographic score, the best condition is defined to have improvement on day 3 when compared with day 1 . Zero score will be assigned to patients who were in the best condition of the corresponding risk factor. The sum of these scores, i.e. the total risk score, represented the overall risk of a case being SARS. The cut-off for a positive diagnosis in the prediction rule was determined by the receiver operating characteristic (ROC) curve.

\section{Statistical analysis}

Chi-squared, unpaired t-tests or Mann-Whitney U-tests were employed whenever appropriate in the univariate analysis. A p-value of $<0.05$ was taken as significant.

\section{RESULTS}

\section{Patient demography and investigation profiles}

A total of 303 patients with unexplained respiratory illness were admitted to the isolation wards during the study period. Of these, eight were excluded as either day 1 or day 3 chest radiographs was missing at the time of analysis. Forty-four patients met the diagnostic criteria for SARS after 2 days (median, interquartile range 1-5), when ribavirin and steroid (methylprednisolone $n=38$, hydrocortisone $n=6$ ) were commenced as standard anti-SARS protocol $[2,11]$.

In the remaining 251 patients (non-SARS group), SARS was finally excluded by RT-PCR and SARS-CoV antibody testing, as described above. Of these, 118 had radiographical evidence of consolidation. Between-group comparison showed no significant difference in age, sex distribution, respiratory symptoms, myalgia, fever, upper respiratory tract or gastrointestinal symptoms between the two non-SARS subgroups, i.e. those with $(n=118)$ and without radiographical consolidation $(n=133),(p>0.5$, data not shown). Although both non-SARS subgroups had a lower frequency of epidemiological link than SARS patients (table 1,72.7\%, $\mathrm{p}<0.001$ for both), those without pneumonia had a higher frequency compared with their counterparts (11.5 versus $2.5 \%$, respectively, $\mathrm{p}=0.01$ ).

Microbiological confirmation of SARS-CoV infection was obtained on all 44 SARS patients (RT-PCR in sputum, nasopharyngeal aspirate, stool or urine, $68.2 \%$; and four antiSARS-CoV IgG, 100\%). In the non-SARS pneumonia group, the putative aetiology for the pneumonia was found in 24 $(20.3 \%)$ patients. The positive sputum microbiology included Mycobacterium tuberculosis $(n=4)$, Haemophilus influenzae $(n=4)$, Staphylococcus aureus $(\mathrm{n}=2)$, Klebsiella pneumoniae $(\mathrm{n}=1)$, Escherichia coli $(\mathrm{n}=1)$ and Pseudomonas aeruginosa $(\mathrm{n}=1)$. Four non-SARS pneumonia patients (including three with positive 


\begin{tabular}{|c|c|c|c|c|}
\hline \multirow[t]{2}{*}{ TABLE 1} & d demograp & s with seve & \multicolumn{2}{|c|}{ iratory syndrome (SARS) and control patients } \\
\hline & SARS & Controls & Odds ratio $(95 \% \mathrm{Cl})^{\#}$ & p-value \\
\hline Subjects $n$ & 44 & 251 & & \\
\hline Mean age yrs & $39.27 \pm 11.26$ & $42.04 \pm 12.49$ & $0.982(0.956-1.008)$ & 0.172 \\
\hline Sex male & 22 & 140 & $0.79(0.42-1.51)$ & 0.478 \\
\hline \multicolumn{5}{|l|}{ Clinical symptoms (day $1-3$ ) } \\
\hline Cough & 19 & 163 & $0.41(0.21-0.79)$ & 0.007 \\
\hline Sputum production & 6 & 97 & $0.25(0.10-0.62)$ & 0.003 \\
\hline Dyspnoea & 6 & 41 & $0.81(0.32-2.04)$ & 0.652 \\
\hline Myalgia & 22 & 75 & $2.35(1.23-4.49)$ & 0.010 \\
\hline Chills & 25 & 99 & $2.02(1.06-3.86)$ & 0.033 \\
\hline Gastrointestinal symptoms & 9 & 44 & $1.21(0.54-2.70)$ & 0.642 \\
\hline \multicolumn{5}{|l|}{ Laboratory parameters } \\
\hline Thrombocytopenia day 1-3 & 17 & 41 & $3.22(1.61-6.45)$ & 0.001 \\
\hline Leukocytosis day $1-3$ & 11 & 85 & $0.65(0.31-1.35)$ & 0.250 \\
\hline Lymphocytopenia day $1-3$ & 38 & 143 & $4.78(1.95-11.72)$ & 0.001 \\
\hline $\begin{array}{l}\text { Elevated alanine transaminase } \\
\text { day } 1-3\end{array}$ & 31 & 103 & $3.43(1.71-6.86)$ & 0.001 \\
\hline \multicolumn{5}{|l|}{$\begin{array}{l}\text { Chest radiographic progress } \\
\text { comparing day } 1 \text { to day } 3\end{array}$} \\
\hline Unchanged & 17 & 178 & $0.26(0.13-0.53)$ & $<0.001$ \\
\hline Deterioration & 26 & 35 & $8.91(4.2-19.07)$ & $<0.001$ \\
\hline
\end{tabular}

Data presented as $\mathrm{n}$ or mean $\pm \mathrm{SD}$, unless otherwise stated. Refer to methods for definitions of epidemiological linkage, clinical symptoms, laboratory parameters and radiographical evaluation. 95\% $\mathrm{Cl}$ : 95\% confidence interval. \#: obtained by comparing SARS with control patients; ": obtained on univariate comparison between SARS patients and controls.

sputum microbiology) had bacteremia, including E. coli $(\mathrm{n}=2)$, K. pneumoniae $(\mathrm{n}=1)$ and $S$. aureus $(\mathrm{n}=1)$. Evidence of respiratory virus infection was documented in nine other patients with non-SARS pneumonia as follows: influenza A $(n=5)$, influenza B $(n=1)$, parainfluenza virus $3(n=2)$ and respiratory syncytial virus $(n=1)$.

\section{Independent risk factor evaluation}

In univariate analysis, seven parameters had positive correlations for SARS: epidemiological link, myalgia, chills, thrombocytopenia, lymphopenia, elevated ALT and chest radiograph progression. Three parameters including cough, sputum production and chest radiograph improvement had negative correlations for SARS. Table 1 shows that SARS patients appear to be more likely to show unchanged versus improvement in radiographical progress than controls (odds ratio (OR) $3.63 ; 95 \%$ confidence interval (CI) 0.47-28.11). Similarly, SARS patients were more likely to have deterioration in radiographical progress than improvement, when compared with controls (OR 28.23, 95\% CI 3.64-219.15).

In the logistic regression analysis, only five of the 14 parameters were found to be independent predictors for SARS. These comprised: a positive epidemiological link (coefficient 5.5; OR 235; 95\% CI 29.1-1895; p<0.0001), myalgia (coefficient 1.4; OR 4.1; 95\% CI 1.4-12.3; p=0.01), lymphopenia between day 1-3 (coefficient 1.6; OR 4.7; 95\% CI 1.2-19.3; $\mathrm{p}=0.03$ ), elevated ALT between day 1-3 (coefficient 1.4; OR 3.8; 95\% CI 1.3-11.5; $\mathrm{p}=0.02$ ), and radiological progression (no change versus improved: coefficient 0.5 ; OR 1.7 ; $95 \%$ CI $0.11-$ 24.3; $\mathrm{p}=0.7$, deteriorated versus improved: coefficient 5.1 ; OR 157; 95\% CI 6.2-3978; $\mathrm{p}=0.002$, deteriorated versus no change: coefficient 4.6 ; OR 94.5; 95\% CI 11.3-792; p<0.0001).

\section{Scoring system for high- or low-risk groups for SARS}

The final model has an adjusted coefficient of determination of 0.73 , which showed reasonably good fit. The following point scores were used to measure the magnitude of the association of each predictor with SARS: epidemiological link, 11 points; myalgia at presentation, 3 points; radiographic deterioration between day $1-3,10$ points and no change, 1 point; lymphopenia between day 1-3, 3 points; and elevated ALT between days $1-3,3$ points. The maximum total risk score is 30 , which is most likely to be a SARS case for any given patient.

The distribution of the total risk score for patients in the SARS and non-SARS groups have been evaluated by a ROC curve and scatter plot (fig. 1 and 2). This prediction rule classified 


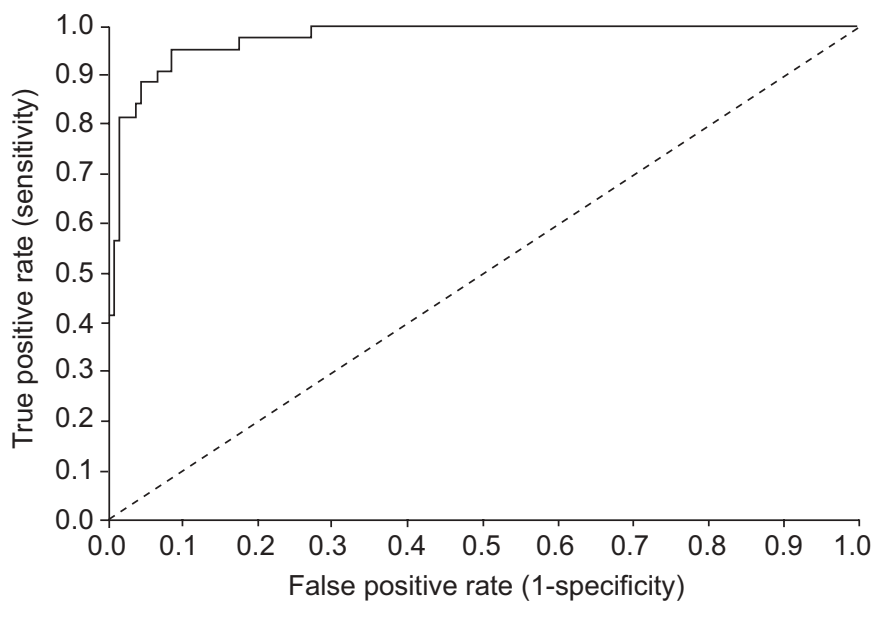

FIGURE 1. Receiver operating characteristic (ROC) plot of the total risk scores for the bedside prediction of severe acute respiratory syndrome. Area under the ROC curve ( \pm SE): $0.96 \pm 0.011$; $95 \%$ confidence interval 0.95-0.99. -----: line of no discrimination.

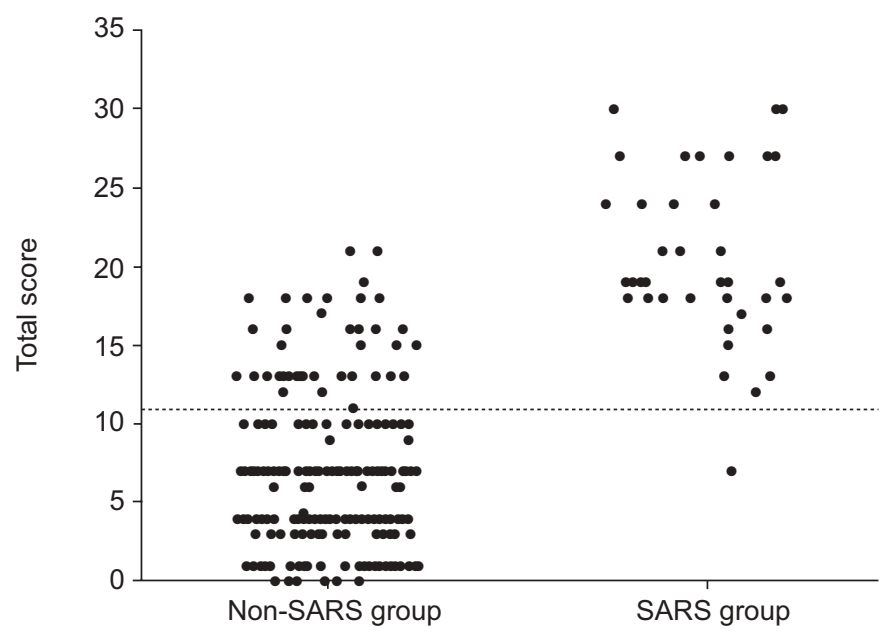

FIGURE 2. Evaluation of the sensitivity and specificity of a prediction rule for the clinical diagnosis of severe acute respiratory syndrome (SARS). Each patient was assigned a total risk score according to the number of independent predictors present. The cut-off line for positive diagnosis was established by the receiver operating characteristic curve.

SARS and non-SARS patients with a ROC curve area $( \pm \mathrm{SE})$ of $0.96 \pm 0.011,95 \%$ CI $0.95-0.99$. Table 2 shows the risk classification into high (score 11-30) and low (score 0-10) risks for SARS, according to the aforementioned prediction model. The sensitivity and specificity of this scoring system in positively identifying a SARS patient are 97.7 and $81.3 \%$, respectively. The positive and negative predictive values are 47.8 and $99.5 \%$, respectively. This model therefore appears to have a very strong power for a negative prediction.

\section{DISCUSSION}

In the cohort of 295 adult patients initially admitted to isolation wards at Queen Mary Hospital with unexplained respiratory illness $[8,16]$, those subsequently diagnosed to have

\begin{tabular}{|c|c|c|c|c|}
\hline TABLE 2 & \multicolumn{4}{|c|}{$\begin{array}{l}\text { Risk classification for severe acute respiratory } \\
\text { syndrome (SARS) according to the bedside } \\
\text { prediction model }\end{array}$} \\
\hline \multicolumn{2}{|c|}{ Risk group for SARS } & Score & SARS & non-SARS \\
\hline \multicolumn{2}{|l|}{ High } & $11-30$ & 43 & 47 \\
\hline \multicolumn{2}{|l|}{ Low } & $0-10$ & 1 & 204 \\
\hline \multicolumn{2}{|l|}{ Total } & & 44 & 251 \\
\hline
\end{tabular}

SARS differed from their counterparts in displaying a positive epidemiological link, myalgia, lymphopenia, elevated ALT, and radiological deterioration versus improvement. The epidemiological link, defined as a history of contact with a SARS patient, or visiting a hospital with nosocomial SARS outbreak, in the 10 days preceding disease onset, is more specific than that proposed by WHO [16]. A component of the latter defined such link as "residing in an area with recent local transmission of SARS" [16], which would have classified the Hong Kong population as having a positive link, and thus was effectively useless for the current authors' practice during the 2003 outbreak. Therefore, the presence of an epidemiological link (11 points) is highly predictive of SARS even in the absence of all the other factors. Conversely, in the absence of an epidemiological link and without the benefit of radiograph observation over the next 2-3 days, the highest possible score on day 1 is 9 points (generated by the presence of myalgia (3 points), lymphopenia (3 points) and elevated ALT (3 points)), and thus inadequate to category a patient into high risk group. Nonetheless, even in the absence of an epidemiological link, the presence of radiographic deterioration and any one of the aforementioned predicting factors could readily classify a patient as high risk for SARS on day 3 . The strength of the prediction model includes the availability of a stringently recruited control group, which is one of the largest $[17,18]$. In addition, most of the SARS patients in the current study were not healthcare workers, as there was a very low staff infection rate (namely two cases altogether who were not recruited), and thus diagnostically more challenging. The prediction rule of the current authors had a high negative predictive value but a low positive one, and thus has such limitation in identifying SARS patients. Nonetheless, it is a useful aid to exclude SARS as a diagnosis in the presence of an unexplained respiratory illness during an outbreak, for clinical management as well as implementation of isolation policy for protection of staff, other patients and the community at large.

The recent four cases in Guangdong, China have vividly shown the difficulties in diagnosing SARS, especially for sporadic cases in a non-outbreak situation [1]. Since March 2003, the WHO and CDC diagnostic criteria have been revised several times, probably due to their low sensitivity (17-36\%) [8, $16,17,19]$. The most updated versions of these diagnostic criteria, requires the presence of clinical symptoms, epidemiological contacts and laboratory evidence of SARS-CoV infection [6, 7]. The criteria on evaluation of symptoms and radiographic appearances are actually unhelpful at the 
bedside. Although there is a wide spectrum of radiological pattern in SARS, including the presence of ground glass opacities, frank consolidation and nodular patterns [11, 20, 21], the current authors have previously shown, albeit qualitatively, that rapid progression is the most characteristic for SARS $[2,5,11]$. Without a gold diagnostic standard, it had been impossible to determine the usefulness of RT-PCR and serological testing for SARS-CoV. Nasopharyngeal aspirate and stool samples could be used for early diagnosis of SARS by detection of genomic RNA using real-time quantitative RTPCR assay for SARS CoV, although the sensitivity is at most $80 \%$ [9]. RT-PCR systems to detect blood or serum SARS-CoV RNA provide a sensitivity of $50-87 \%$ during the first week of illness, but drop to $25 \%$ at day 14 after fever onset [10]. Bedside diagnosis of SARS, therefore, remains a key process in the management of a patient suspected to have SARS.

Two scoring systems, designed to demarcate severe acute respiratory syndrome from other patients, have been developed. Data derived from 232 patients were used to derive a scoring system whereby attribution of $3,3,2,1,1,1$ and 1 points was made to the presence of multilobar radiographic infiltrates, sputum monocyte predominance, lymphopenia, epidemiological linkage, high lactate dehydrogenase, high Creactive protein, and prolonged activated partial prothrombin time, respectively. At a cut-off of 6 points, the sensitivity and specificity in diagnosing severe acute respiratory syndrome were reported as 100 and 93\% [17]. Another system, derived from data on eight probable severe acute respiratory syndrome cases out of 70 patients presenting to an emergency department, showed a 6-item scoring system based on the presence of cough, myalgia, diarrhoea, rhinorrhoea or sore throat, lymphopenia, thrombocytopenia. This was reported to detect severe acute respiratory syndrome with $100 \%$ sensitivity and $86.3 \%$ specificity [18]. While these systems appear to be simple and user-friendly, they unfortunately lack control data to support their validity. In addition, these authors have not stringently defined the selection criteria thus restricting the extrapolation of their findings to other centres. While the current study has a control group, it suffers from a small sample size and a lack of validation as it is only derived from one centre and thus could be potentially biased and not applicable to other populations. The effects of variation in the prevalence during the outbreak, which could have affected the predictive values of the model, were not evaluated. These effects could potentially affect the clinical application of the model and should therefore be addressed in future studies. The validity of the current authors' prediction model should also be evaluated in other cohorts, including those in Hong Kong, mainland China, Singapore, Toronto and Taiwan. It is also important to test its validity in the form of sensitivity and specificity should severe acute respiratory syndrome recur.

\section{ACKNOWLEDGEMENTS}

The authors would like to thank A. Wong, W. Wong, and P. Lee for their invaluable assistance in collecting data prospectively directly from the severe acute respiratory syndrome patients at Queen Mary Hospital, Hong Kong SAR, China.

\section{REFERENCES}

1 World Health Organization. WHO calls for urgent investigation into sources of infection in recent SARS cases in Guangdong Province, after a fourth case is announced. January 31, 2004. www.wpro.who.int/sars/ docs/pressreleases/pr_31012004.asp. Date updated: 31 January 2004; Date accessed: 18 June 2005.

2 Tsang KW, Ho PL, Ooi GC, et al. A cluster of cases of severe acute respiratory syndrome in Hong Kong. $N$ Engl J Med 2003; 348: 1977-1985.

3 Hsu LY, Lee CC, Green JA, et al. Severe acute respiratory syndrome (SARS) in Singapore: clinical features of index patient and initial contacts. Emerg Infect Dis 2003; 9: 713-717.

4 Poutanen SM, Low DE, Henry B, et al. Identification of acute respiratory syndrome in Canada. N Eng J Med 2003; 348: 1995-2005.

5 Peiris JS, Chu CM, Cheng VC, et al. Clinical presentation and viral load in a community outbreak of coronavirusassociated SARS pneumonia: a prospective study. Lancet 2003; 361: 1767-1772.

6 World Health Organization. Case definitions for surveillance of severe acute respiratory syndrome (SARS). www.who.int/csr/sars/casedefinition/en/. Date updated: 1 May 2003; Date accessed: 18 June 2005.

7 Center for disease prevention and control. Severe acute respiratory syndrome (SARS). Diagnosis/evaluation. www.cdc.gov/ncidod/sars/diagnosis.htm

8 Centers for Disease Control and Prevention. March 2003. www.cdc.gov/ncidod/sars/. Date updated: 3 May 2005; Date accessed: 18 June 2005.

9 Poon LL, Chan KH, Wong OK, et al. Detection of SARS coronavirus in patients with severe acute respiratory syndrome by conventional and real-time quantitative reverse transcription-PCR assays. Clin Chem 2004; 50: $67-72$

$10 \mathrm{Ng}$ EK, Hui DS, Chan KC, et al. Quantitative analysis and prognostic implication of SARS coronavirus RNA in the plasma and serum of patients with severe acute respiratory syndrome. Clin Chem 2003; 49: 1976-1980.

11 Tsang KW, Lam WK. Management of severe acute respiratory syndrome: the Hong Kong University experience. Am J Respir Crit Care Med 2003; 168: 417-424.

12 Ho JC, Ooi GC, Mok TY, et al. High-dose pulse versus nonpulse corticosteroid regimens in severe acute respiratory syndrome. Am J Respir Crit Care Med 2003; 168: 1449-1456.

13 Ooi CG, Khong PL, Lam B, et al. Severe acute respiratory syndrome: relationship between radiologic and clinical parameters. Radiology 2003; 229: 492-499.

14 Agresti A. Models for binary response variables. In: Categorical Data Analysis. New York, Wiley, 1990; pp. 79-129.

15 Fine MJ, Auble TE, Yealy DM, et al. A prediction rule to identify low-risk patients with community-acquired pneumonia. N Engl J Med 1997; 336: 243-250.

16 World Health Organization. March 2003. Case definitions for surveillance of severe acute respiratory syndrome (SARS) 2003. www.who.int/csr/sars/casedefinition. Date updated: 1 May 2003; Date accessed: 18 June 2005. 
17 Wang TL, Jang TN, Huang CH, et al. Establishing a clinical decision rule of severe acute respiratory syndrome at the emergency department. Ann Emerg Med 2004; 43: 17-22.

18 Chen SY, Su CP, Ma MH, et al. Predictive model of diagnosing probable cases of severe acute respiratory syndrome in febrile patients with exposure risk. Ann Emerg Med 2004; 43: 1-5.

19 Rainer TH, Cameron PA, Smit D, et al. Evaluation of WHO criteria for identifying patients with severe acute respiratory syndrome out of hospital: prospective observational study. BMJ 2003; 326: 1354-1358.

20 Ooi CG, Khong PL, Ho JC, et al. Severe acute respiratory syndrome: radiographic evaluation and clinical outcome measures. Radiology 2003; 229: 500-506.

21 Muller NL, Ooi GC, Khong PL, Zhou LJ, Tsang KW, Nicolaou S. High-resolution CT findings of severe acute respiratory syndrome at presentation and after admission. Am J Roentgenol 2004; 182: 39-44. 